\title{
Identifying the Dynamics of Leg Muscle Activation During Human Gait Using Neural Oscillator and Fuzzy Compensator
}

\author{
Reihaneh Ravari, Hamid Reza Kobravi* \\ Research Center of Biomedical Engineering, Islamic Azad University of Mashhad, Mashhad, Iran
}

\begin{abstract}
Background: The goal of this study is to design a model in order to predict the muscle activation pattern because the muscle activation patterns contain valuable information about the muscle dynamics and movement patterns. Therefore, the goal of the presentation of this neural model is to identify the desired muscle activation patterns by Hopf chaotic oscillator during walking. Since the knee muscles activation has a significant effect on the movement pattern during walking, the main concentration of this study is to identify the knee muscles activation dynamics using a modeling technique.

Methods: The electromyography (EMG) recording obtained from 5 healthy subjects that electrodes positioned on the tibialis-anterior (TA) and rectus femoris muscles on every 2 feet. In the proposed model, along with the chaotic oscillator, a fuzzy compensator was designed to face the unmolded dynamics. In fact, on the condition, the observed difference between the desired and actual activation patterns violate some specific quantitative ranges, the fuzzy compensator based on predefined rules modify the activity pattern produced by the Hopf oscillator.

Results: Some quantitative measures used to evaluate the results. According to the achieved results, the proposed model could generate the trajectories, dynamics of which are similar to the muscle activation dynamics of the studied muscles. In this model, the generated activity pattern by the proposed model cannot follow the desired activity of the TA muscle as well as rectus femoris muscle.

Conclusion: The similarity between the generated activity pattern by the model and the activation dynamics of Rectus- Femoris muscle was more in comparison with the similarity observed between activation pattern of Tibialis- Anterior and the pattern generated by the model. In other words, based on the recorded human data, the activation pattern of the Rectus- Femoris is more similar to a rhythmic pattern.
\end{abstract}

Keywords: Muscle activation pattern; Fuzzy logic system; Hopf oscillator; Gait analysis.

\author{
* Correspondence to \\ Hamid Reza Kobravi, \\ Research Center of Biomedical \\ Engineering, Mashhad Branch, \\ Islamic Azad University, \\ Mashhad, Iran.
}

Email: hkobravi@mshdiau.ac.ir

Published online 30 September 2018

Citation: Ravari R, Kobravi HR. Identifying the dynamics of leg muscle activation during human gait using neural oscillator and fuzzy compensator. Int Clin Neurosci J. 2018;5(3):106-112. doi:10.15171/icnj.2018.21

\section{Introduction}

The electromyography (EMG) signal measures the elicited electrical currents generated during the muscle contraction representing the neuromuscular activities. The recorded surface EMG signals show the motor neurons activity, can reflect the relative level of muscle activation and bear valuable information about muscle functions. ${ }^{1,2}$ The nervous system controls muscle activities such as contraction and relaxation. Hence, the complicated EMG signal, which is controlled by the nervous system, is dependent on the anatomical and physiological properties of muscles. ${ }^{3}$ The EMG recordings have shown that the activity patterns of the various involved muscles during the locomotion, may exhibit considerable stepby-step variability. In other words, an electromyographic activity pattern of each involved muscle during the gait cycle exhibits a considerable intersubject, intermuscle and context-dependent variability. ${ }^{4}$ The EMG signals can use for clinical and biomedical applications, evolvable hardware chip (EHW) development, and modern humancomputer interaction. ${ }^{3}$ The researchers significantly address the field of motor rehabilitation, as an application area.

It has shown recently that the nervous system can produce different movements by controlling the muscles by activating flexible combinations of muscle synergies. In other words, though the many muscles may involve during the locomotion, that human locomotion can control by flexible combinations of a small number of muscle activity patterns. ${ }^{5}$ The use of such models has

(C) 2018 The Author(s). This is an open access article distributed under the terms of the Creative Commons Attribution License (http:// creativecommons.org/licenses/by/4.0/), which permits unrestricted use, distribution, and reproduction in any medium, provided the original work is properly cited. 
limitations, such as generalizability and repeatability for different individuals in different situations.

The reproducible pattern of muscle activation during walking in healthy adults has observed. Such pattern generated due to inhibition of gastrocnemius/soleus (GS/ SOL) muscle activity followed by activation of the tibialisanterior (TA) muscle bilaterally. A temporally invariant relationship between soleus muscle (SOL) inhibition and TA activation has found which is utilized by the central nervous system (CNS) as a motor program for controlling the gait initiation. ${ }^{6}$ Also, the coordinated hip muscle activities accelerate the center of mass (COM) movement and allow the swing limb to be elevated. ${ }^{7-9}$ Thus, from the mechanic's point of view undermining the coordination of muscle groups is an important issue. ${ }^{10}$

In recent studies, the EMG of residual limb muscles of amputees may bear useful information on adaptations besides those that can be found using the kinetic data. ${ }^{11}$ It has reported that the co-activation of the upper leg muscles of the intact limb in amputees was greater than in controls. ${ }^{12}$ Furthermore, researchers reported that during the gait, the muscle activity of residual limb muscles in three trans-femoral amputees correlates with the socket pressure. ${ }^{13}$ Muscle activity per gait phase (stance and swing) can give more insight to the changes are observed in the muscle activity patterns in amputees. ${ }^{14}$

Some researchers showed that three basic patterns could account for the activity of some leg muscles involved during the locomotion. Accordingly, it has suggested that the observed complex muscle activity patterns may control by a few underlying activity pattern generators. ${ }^{15,16}$

In recent researches, muscle activation in non-disabled participants investigated by recording surface EMG during treadmill walking at different speeds. They observed that each muscle had a different activation pattern throughout the gait cycle. With increasing walking speed, the phasedependent activation remained similar, but the mean amplitudes generally increased. ${ }^{3}$ The recent studies show significant differences in both spatial and temporal properties of the most muscles of the lower extremity. The compared muscle activation patterns measured during ambulating within a commercially available robotic orthosis. The researchers hypothesize that after walking using the robotic orthosis for just a short period; subjects could adapt their gait pattern and learn a new set of motor commands necessary to carry out the restricted movement imposed by the robot. Since the device imposes numerous restrictions on the gait pattern, some differences in the activation patterns of some muscles found. ${ }^{17}$

In previous studies, a neural network has been proposed to predict the muscle activations using EMG signals [18]. Using a 4-layer feed-forward neural network to predict the muscle activations avoids establishing a complex mathematical model to express the muscle activation dynamics. In this study, the basic back-propagation algorithm was not applicable, because the real muscle activation was not available. ${ }^{18}$ Once the muscle activations obtained, the muscle force was estimated using the Hilltype models. ${ }^{18}$

Some certain points obtained from last studies that present different musculoskeletal models for predicting muscle activity patterns. Besides, the musculoskeletal modeling can be used to calculate many significant kinetic data that are difficult to be measured such as joint force or ligament force. ${ }^{19}$

In previous papers, a musculoskeletal model of the lower limb is used to predict the hip contact forces and elicited muscle activations during walking at different speeds for three total hip replacement patients implanted with instrumented prostheses. The developed model could estimate the magnitude of hip contact forces with encouraging accuracy regarding relative peak error. As documented in the literature, the predictions were more accurate for slow walking speed. The static optimization technique adopted to estimate muscle activation profiles reproduced for the majority of muscles for different walking speeds. ${ }^{20,21}$ The other model's study shows that, this model, which was optimized based on the EMG driven model, as an EMG-driven model can predict unseen trials. Researchers in this study evaluated this optimization model on 2 stroke patients. The corrective changes in EMG patterns of muscles during the walking trials observed. These calculated corrective changes could use as reference data used for the stroke patients' gait training by functional electrical stimulation (FES). According to the reported results, the TA activity during swing phase in post-stroke subjects was similar to that found in unimpaired subjects. Also, the expected second peak of TA activity at initial foot contact lacked. This expected peak of activity might account for their flat foot walking. Thus, correcting the Tibialis- Anterior activity is needed to avoid the drop-foot. ${ }^{22,23}$

The aim of this study according to the previous works that had done, is to present a model using the chaotic oscillator, which can generate the desired activity pattern. In this study, the use of chaotic oscillator is so essential, because EMG has chaotic nature. According to this issue that stimulation pattern is a multiple of activity pattern, using activity pattern yielded the desired stimulation pattern. The desired stimulation pattern can help dropfoot patients to restore their ability to walk. In other words, the purpose of this study is to present a model that generates the desired muscle activity pattern.

\section{Materials and Methods \\ Data Collection}

In this study, the identification of the activity pattern's model based on real experimental recorded data. Therefore, at the first step, EMG data recorded from 5 healthy subjects who were 2 males and three females between the ages of 26-34 years. In this experiment, the limitation for the stride length and walking speed does not 
consider, and all the subjects walked with preferred speed. Chest leads located on the TA and rectus- femoris muscles. Every subject walked 20 steps during recording the data. The device of recording EMG signal is Flexcomp. The Flexcomp is a 10-channel recording system. It as a multimodality encoder has some needed flexibilities for realtime, computerized biofeedback and data acquisition in the different clinical setting. The Flexcomp covers the full range of objective physiological signals used in research and clinical applications. It has software that installed on the computer which facilitates easy data recording. EMG recording electrodes positioned on the TA and rectusfemoris muscles on every 2 feet. Researchers focused on surface electromygraphy (sEMG) analyses can appreciate its ability to monitor, to record and to display the raw sEMG. Besides, computing and displaying the median frequency, RMS, peak-to-peak, 2D and 3D frequency and power spectrum of the signals is possible.

The type of electrodes used was Ag-AgCl. Good electrical contact achieved between the recording amplifier and the muscles. In order to extract the desired activity pattern from the EMG signals, the main signal passed from bandpass filter with order 3 and butter worth filter to noise elimination. At the same time with the EMG recording, angle changes of people's joint during walking were obtained, for which we can write fuzzy rules basis. In other words, using recorded data from healthy subjects and the range of angle joints changes of the foot during walking in the subjects, fuzzy rules were obtained. Writing the fuzzy rules are based on the changes of foot's joints and the membership function of their changes (Figure 1).

In this study, the differences between the actual angle and the desired angle are the input of the fuzzy system, and the desired muscle activity pattern is the output of the fuzzy system.

\section{The Proposed Model}

In this study, the Hopf oscillator generated an activity pattern. The Hopf oscillator has used as a trained pattern generator. According to some reported studies, ${ }^{24}$ observing chaotic nature in the behavior of the EMG signal is not surprising. Therefore, the Hopf oscillator as an adaptive oscillator was utilized, which can potentially generate chaotic dynamics. ${ }^{24}$

Although the oscillator was trained using human data, the time-varying dynamic of gait may undermine the performance of the oscillator to track the dynamic

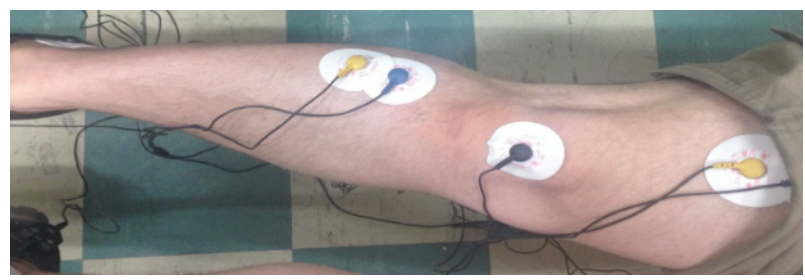

Figure 1. Placement of the electrodes. variations accurately. So a fuzzy compensator was added to cope with this problem. The fuzzy rules designed in a manner that can modify the muscle activation pattern generated by the oscillator during the time. The fuzzy logic system corrects the activation level whenever the ankle joint angle violates the specific boundaries, which must restrict within them during each gait phase. In other words, the differences between the actual angle and the joint angle trajectory obtained using the recorded data related to the healthy subjects were the input of the fuzzy system. Based on the designed rules, the necessary changes given on the oscillator generated pattern. It means that according to the difference between the desired pattern and the pattern generated by the oscillator, the fuzzy compensator modifies the generated pattern by the Hopf oscillator in order to be closer to the desired pattern.

Figure 2 shows the structure of the proposed model. In Figure 2, $\Delta$ as the input of the fuzzy system is the difference between the actual angle and the extremes of the desired angle profile related to the healthy subject. $U c$ is also the output of the fuzzy system which generated through some designed fuzzy rules. $U_{1}$ is also the generated pattern by the Hopf oscillator. $U c$ is a compensatory component modifying the output of HOPF oscillator during the gait cycle.

\section{The HOPF Oscillator}

In recent years, central pattern generators (CPGs) models are progressively used to control the vast kinds of autonomous robots' locomotion. ${ }^{25-28}$ CPGs often modeled through coupled nonlinear oscillators. ${ }^{29}$ The Complex oscillatory patterns can be generated using the coupled nonlinear oscillators. Hence, it will make these systems fascinating in order to such models can be used to model animals' gaits and to control robots. ${ }^{30}$ However, since the different parameters have to be tuned by an optimization algorithm or by hand, in most cases the design of such CPGs is not trivial. Indeed, the values of different parameters determining the frequency of the oscillations and their respective phase lags, usually need

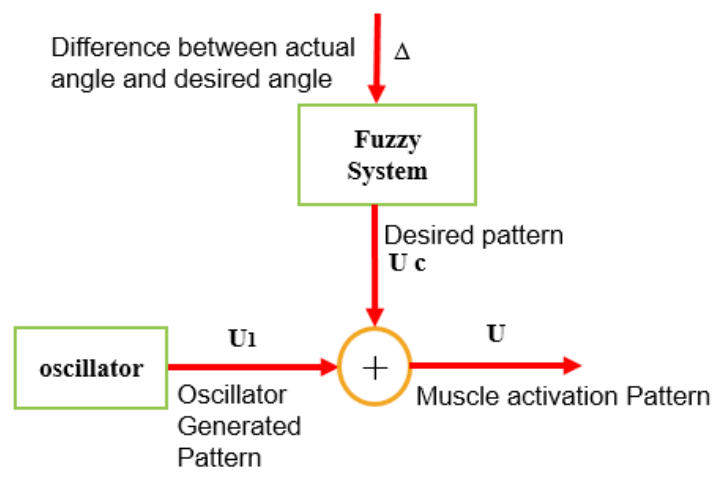

Figure 2. Block Diagram of the Desired Activity Pattern Generated. 
to be adjusted. ${ }^{24}$

In this study, the coupled Hopf oscillator used. The purpose of such a coupling is to keep the correct phase differences between the oscillators. ${ }^{24}$ As shown in Figure 3 , in the coupled Hopf oscillator, a phase signal $\mathrm{Ri}$, which is in-phase with $\mathrm{x} 0$, has the frequency of oscillator $i$. If we couple Ri with oscillator I, then it will be seen phaselocking between oscillator 0 and i. Each oscillator receives the same learning signal $\mathrm{F}(\mathrm{t})=\mathrm{P}_{\text {teach }}(\mathrm{t})-$ - aixi which is the difference between the signal to be learned, $\mathrm{P}_{\text {teach }}(\mathrm{t})$, and the signal already learned, $Q_{\text {learned }}(t)$. Then all the oscillators (except oscillator 0 ) receive the scaled phase input Ri from oscillator 0 .

The equation for oscillator $i$, with coupling to oscillator 0 for phase-lock, is given by the following equations:

$$
\begin{aligned}
& \dot{x}_{i}=\gamma\left(\mu-r_{i}{ }^{2}\right) x_{i}-\omega_{i} y_{i}+\varepsilon \mathrm{F}(\mathrm{t})+\tau \sin \left(R_{i}\right) \\
& \dot{y}_{l}=\gamma\left(\mu-r_{i}{ }^{2}\right) y_{i}+\omega_{i} x_{i}
\end{aligned}
$$

Where $x_{i}, y_{i}$, and $\omega_{i}$ describe the $i_{\text {th }}$ adaptive Hopf oscillator. $r_{i}=\sqrt{x^{2}+y^{2}}$ and $\varepsilon$ are positive coupling constants controlling the learning rate, and $\tau$ is a coupling constant. Coupling all the oscillators with oscillator 0 assure stable phase-locked oscillations. ${ }^{31}$

$\tau$ is a coupling constant and $r_{i}=\sqrt{x^{2}+y^{2}}$ and $\varepsilon$ are positive coupling constants controlling the learning rate. All employed oscillators coupled with oscillator 0 . Such couplings give assurance of stability of phase-locked oscillations. $^{31}$

\section{Designed Fuzzy Logic System}

Fuzzy logic is a kind of logic that not only includes true or false values; also it uses a continuous range of truth values in the interval $[0,1]$. Furthermore, it lets us combine numerical data and linguistic knowledge systematically. ${ }^{32}$ Fuzzy systems based on human knowledge in the form of if-then rules. Mamdani fuzzy system has used in this study. The general structure of fuzzy rules in this research is as follows:

\section{If $\theta_{\mathrm{d}}$ is $\mu_{A}$ and $\Delta$ is $\mu_{B}$ then $\mu_{C}$ is $\mu_{C}$.}

In the fuzzy rules, $\theta_{d}$ desired angle of the ankle in the healthy subjects, $\Delta$ is the angle difference of the ankle in the healthy subjects and patients and $\mu_{C}$ as the output of fuzzy logic system determines the compensatory signal correcting the muscle activity pattern generated by HOPF oscillator activity derived from healthy individuals. Finally, some membership functions assigned for $\theta_{d}, \Delta$, and $\mu_{C}\left(\mu_{A}, \mu_{B}, \mu_{C}\right)$ (Figure 4 ).

The following lookup table shows the designed fuzzy rules. According to this table, the rules with less importance eliminated. The rules were extracted using analyzing the range of recorded human data related to ankle joint movement. At first, the range of ankle joint motion during each gait phase was specified using healthy human data analyses. Then, the fuzzy rules were designed to adjust the output of the HOPF oscillator according

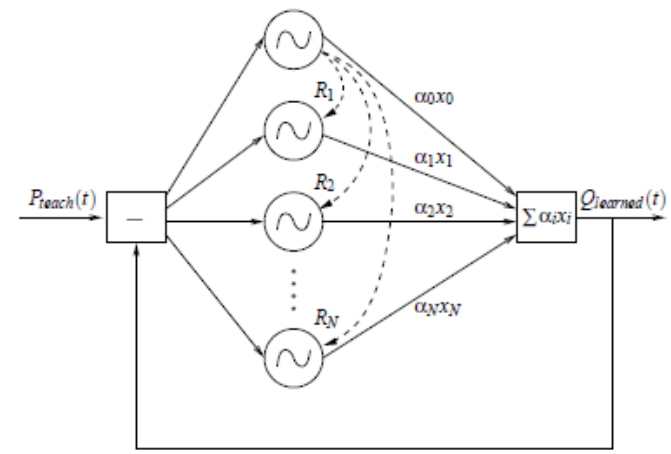

Figure 3. The Adaptive Hopf Coupled Oscillator.

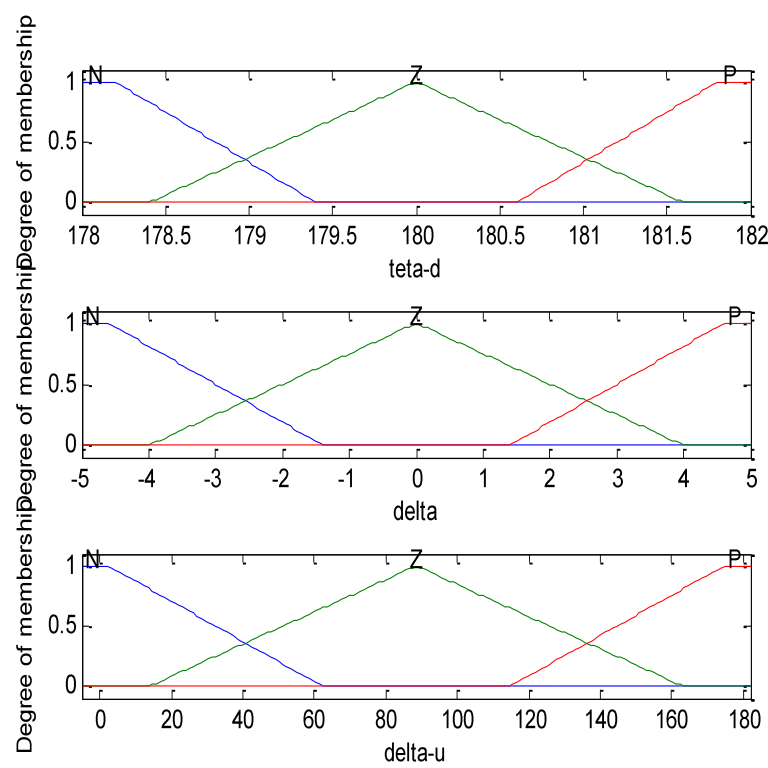

Figure 4. The Membership Function Of Fuzzy Rules.

to the difference between the actual joint angle and the specified range corresponding to that sample time. The fuzzy system as a compensatory controller corrects the muscle activation pattern whenever the ankle joint violates the specific extremes. This correcting process by the fuzzy logic system is carried out in an online manner to reduce the possible difference between the muscle activation pattern obtained using healthy human data (Figure 5).

\section{Results}

Figures 6 and 7 show three sample obtained results. Figure 6 and 7 showed the model outputs along with the extracted Rectus- Femoris muscle activation pattern and extracted Tibialis- Anterior muscle activation pattern respectively. Figure 6 shows the model output while the fuzzy compensatory system has adopted and Figure 7 shows the model output while no fuzzy compensatory system has adopted. The activation patterns extracted from actual data recorded from the healthy subjects 


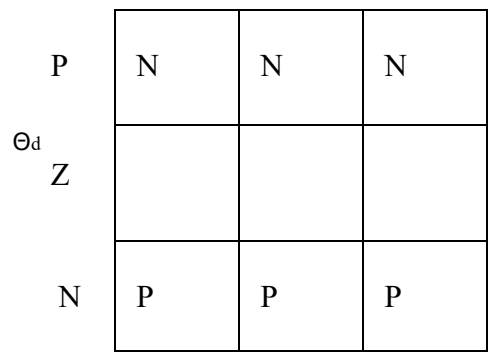

Figure 5. Look-up Table Illustration of Fuzzy Rule-Based.

during walking. It took 20 steps to walk during each trial.

For the sake of quantitative assessment, the correlation coefficient and root mean square error (RMSE) computed. The correlation coefficient, which is the covariance of the variables divided by the product of their standard deviations, between 2 variables $\mathrm{X}$ and $\mathrm{Y}$ can be computed using the following equation:

$\operatorname{Corr}(X, Y)=\frac{\operatorname{cov}(X, Y)}{\delta_{X} \delta_{Y}}$

Where cov means covariance, Corr is the usual symbol for correlation and sigma is the standard deviation symbol.

The root mean square error (RMSE) is a frequently used measure of the difference between values predicted by a model and the values observed from the environment that modeled, and obtained from the following equation:

$\mathrm{RMSE}=\sqrt{\frac{\sum_{i=1}^{n}\left(x_{i}-\dot{x}_{i}\right)^{2}}{n}}$

Where $x_{i}$ is the measured value, $\dot{x}_{i}$ is the predicted value, and $\mathrm{n}$ is the number of data samples.

According to the results, the generated activity pattern by the proposed model cannot follow the desired activity of the Tibialis- Anterior muscle as well as rectus-femoris muscle. The Tables 1 and 2 show the mean value and standard deviation of the computed correlation coefficient and RMSE related to each muscle in all subjects.

The computed quantitative measures imply that the proposed model has had better performance in estimating the rectus- femoris muscle activity.

In the next step, the effectiveness of the utilized compensatory fuzzy system assessed. The performance of the HOPF oscillator lonely and without adding the fuzzy compensatory system assessed.

In Tables 3 and 4, the mean and standard deviation values of the computed correlation coefficient and the RMSE related to each muscle in each subject shown, while no fuzzy compensatory system has used. Tables 3 and 4 elucidate that the model performance has decayed. The achieved results elucidate the importance of the designed fuzzy compensator. Therefore, adding a fuzzy
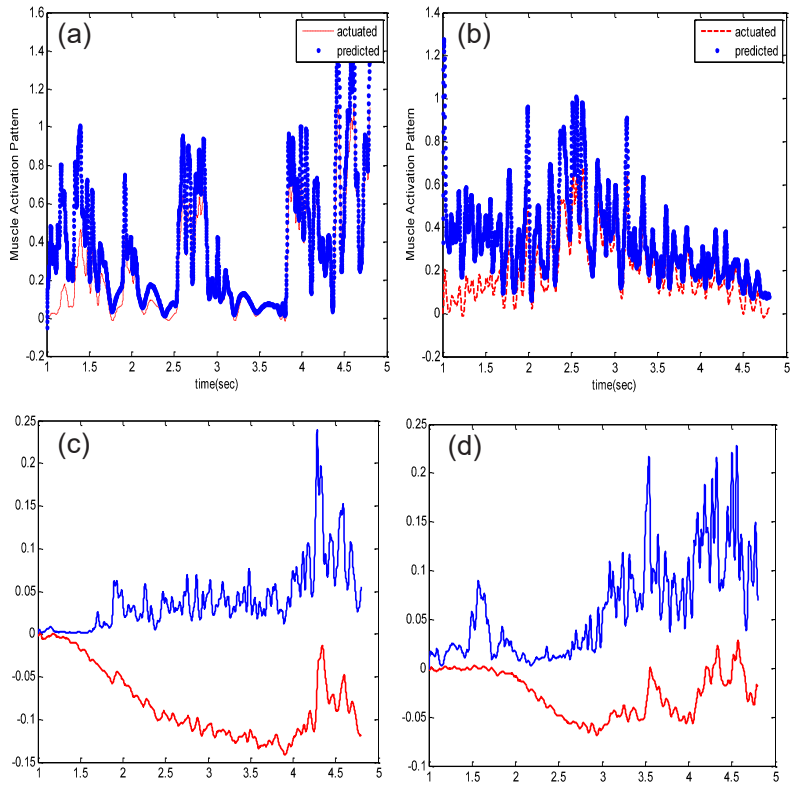

Figure 6. The Desired Activation Pattern of the Rectus- Femoris Muscle (a,b), and Tibialis-Anterior Muscle (c,d), in a Sample Healthy Subject Along With the Activation Pattern Generated by the Hopf Oscillator and a Fuzzy Compensator. (a)
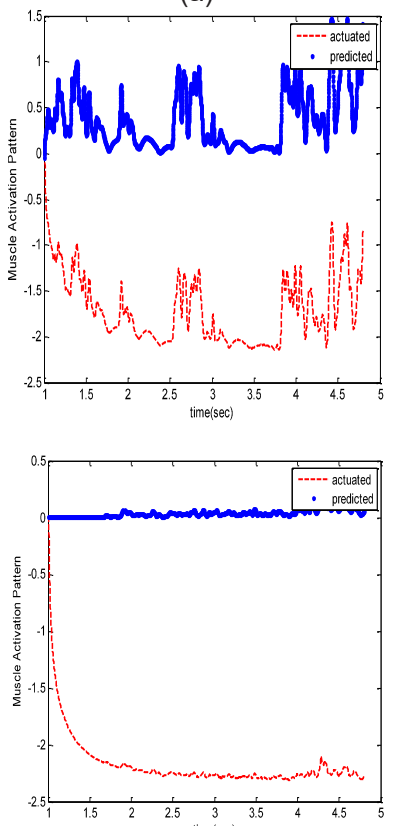

(c) (b)
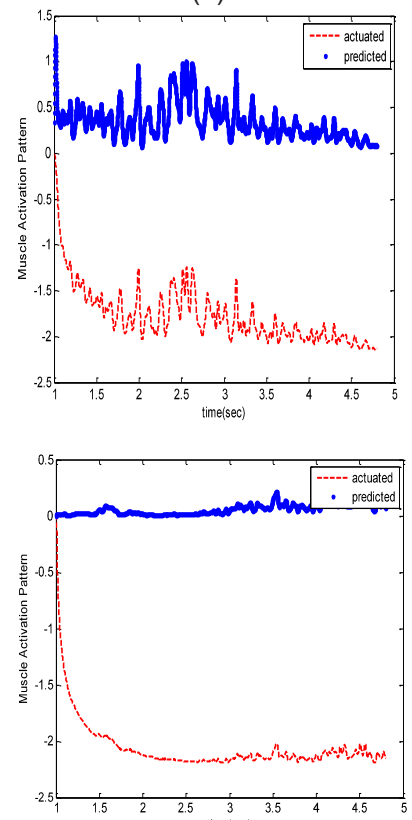

(d)
Figure 6. The Desired Activation Pattern of the Rectus- Femoris Muscle $(a, b)$, and Tibialis-Anterior Muscle (c,d), in a Sample Healthy Subject Along With the Activation Pattern Generated by the Hopf Oscillator Without Using a Fuzzy Compensator.

compensator to cope with the unmolded dynamics seems to be necessary for the proposed model. Such results can ratify what Figures 6 and 7 intuitively demonstrate.

It can conclude that the proposed model, not only with but also without using the added fuzzy compensator, has 
Table 1. The Mean (SD) and Computed Correlation Coefficient Related to Each Subject With Fuzzy Compensator

\begin{tabular}{llllll}
\hline & Subject1 & Subject2 & Subject3 & Subject4 & Subject5 \\
\hline Rectus-femuris & $0.59 \pm 0.19$ & $0.58 \pm 0.17$ & $0.65 \pm 0.25$ & $0.65 \pm 0.13$ & $0.61 \pm 0.30$ \\
Tibialis-anterior & $0.52 \pm 0.17$ & $0.45 \pm 0.14$ & $0.61 \pm 0.08$ & $0.36 \pm 0.32$ & $0.34 \pm 0.20$ \\
\hline
\end{tabular}

Table 2. The Mean (SD) and Computed RMSE Related to Each Subject With Fuzzy Compensator

\begin{tabular}{llllll}
\hline & Subject1 & Subject2 & Subject3 & Subject4 & Subject5 \\
\hline Rectus-femuris & $0.72 \pm 0.97$ & $0.30 \pm 0.24$ & $0.30 \pm 0.14$ & $0.27 \pm 0.17$ & $0.28 \pm 0.15$ \\
Tibialis-anterior & $0.82 \pm 0.85$ & $0.34 \pm 0.20$ & $0.51 \pm 0.33$ & $0.54 \pm 0.32$ & $0.33 \pm 0.19$ \\
\hline
\end{tabular}

Table 3. The Mean (SD) and Computed RMSE Related to Each Subject Without Fuzzy Compensator

\begin{tabular}{llllll}
\hline & Subject1 & Subject2 & Subject3 & Subject4 & Subject5 \\
\hline Rectus-femuris & $0.50 \pm 0.28$ & $0.20 \pm 0.24$ & $0.53 \pm 0.33$ & $0.34 \pm 0.27$ & $0.33 \pm 0.25$ \\
Tibialis-anterior & $0.14 \pm 0.28$ & $-0.03 \pm 0.20$ & $0.24 \pm 0.26$ & $-0.09 \pm 0.22$ & $-0.03 \pm 0.20$ \\
\hline
\end{tabular}

Table 4. The Mean (SD) and Computed RMSE Related to Each Subject Without Fuzzy Compensator

\begin{tabular}{llllll}
\hline & Subject1 & Subject2 & Subject3 & Subject4 & Subject5 \\
\hline Rectus-femuris & $2.10 \pm 0.02$ & $2.12 \pm 0.05$ & $2.16 \pm 0.03$ & $2.13 \pm 0.03$ & $2.12 \pm 0.03$ \\
Tibialis-anterior & $2.12 \pm 0.02$ & $2.16 \pm 0.03$ & $2.15 \pm 0.03$ & $2.14 \pm 0.01$ & $2.15 \pm 0.24$ \\
\hline
\end{tabular}

had better performance in estimating the rectus-femoris muscle activation pattern. Such results can attribute to the apparent similarity between the Hopf oscillators' dynamic and the dynamics of the rectus-femoris muscle activity. It may be because of the rhythmic behavior of the rectusfemoris muscle activity during the gait.

It observed that adding a constant value (about 2) to the output value of the fuzzy compensatory system could nearly improve the model performance. It can be attributed to the shortcoming of the fuzzy compensatory system to overcome the errors emanating from DC drift between the desired muscle activation pattern and model output.

\section{Discussion and Conclusion}

Functional electrical stimulation is one of the well-known solutions applied for gait correction in patients with drop-foot. One of the main restrictions of FES, which limit the prevalence of FES clinical applications, is the online optimization of muscle stimulation profile. One recently raised idea is designing the stimulation profile using muscle activation profile of a healthy person. ${ }^{33} \mathrm{~A}$ complementary idea is designing the stimulation pattern of a paralyzed muscle using muscle activation pattern of the corresponding muscle of contralateral limb; This is a plausible idea, but time-varying properties of muscles may undermine the performance of an FES system with time-invariant stimulation pattern. Accordingly, utilizing an adaptive model generating the desired muscle activation pattern in an online manner can be a good idea. In this study, the adaptive neural oscillator has adopted as the core of the model. However, a compensatory part was added to improve the model performance based on comparing the joint movement pattern with the appropriate laws of the model parameters designed. The kinematic information of the joint was incorporated to update the model instead of model output error. In this manner, adaptation laws update the compensatory part parameters in a way that generated muscle activation pattern, as the input of the muscle-joint system, is adjusted by considering the muscle-joint output dynamics; This is an innovative attitude in parameter optimization. Since in practical uses of rehabilitation systems the joint movements, showing the elicited muscle-joint dynamics, may be disturbed by external disturbance or changing the context, such updating process can cope with the factors destabilizing the mechanical stability of the human movement. Accordingly, using such a model, someone can implement the FES systems with adaptable stimulation pattern, stimulation pattern of which optimized in an online manner.

One intriguing achieved result shows better performance of the designed model in tracking the activation pattern of the rectus-femoris muscle in comparison with the TA; This can lie in this fact that when an oscillator is the core of such a model, the dynamics of the model output is more similar to a rhythmic dynamics. According to the recorded human data, the activation pattern of the rectus- 
femoris is more similar to a rhythmic pattern and can certify the results.

The achieved results prove that such a proposed model may improve the performance of FES systems provided that the stimulation pattern extracted from determined muscle activation pattern through a suitable transformation. Naturally, the next step of this research is the practical implementation of an FES system based on the proposed model.

\section{Conflict of Interest Disclosures}

The authors declare that they have no conflict of interests.

\section{Ethical Statement}

Not applicable.

\section{References}

1. Kleissen RF, Buurke JH, Harlaar J, Zilvold G. Electromyography in the biomechanical analysis of human movement and its clinical application. Gait Posture. 1998;8(2):143-58.

2. Raez MB, Hussain MS, Mohd-Yasin F. Techniques of EMG signal analysis: detection, processing, classification and applications. Biol Proced Online. 2006;8:11-35. doi: 10.1251/bpo115.

3. Ma C, Chen N, Mao Y, Huang D, Song R, Li L. Alterations of Muscle Activation Pattern in Stroke Survivors during Obstacle Crossing. Front Neurol. 2017;8:70. doi: 10.3389/ fneur.2017.00070.

4. Ivanenko YP, Poppele RE, Lacquaniti F. Motor control programs and walking. Neuroscientist. 2006;12(4):339-348. doi: 10.1177/1073858406287987

5. d'Avella A, Portone A, Fernandez L, Lacquaniti F. Control of fast-reaching movements by muscle synergy combinations. J Neurosci. 2006;26(30):7791-810. doi: 10.1523/ jneurosci.0830-06.2006.

6. Crenna P, Frigo C. A motor programme for the initiation of forward-oriented movements in humans. J Physiol. 1991;437:635-53.

7. Winter DA. Human balance and posture control during standing and walking. Gait Posture. 1995;3(4):193-214. doi: 10.1016/0966-6362(96)82849-9.

8. Hass CJ, Gregor RJ, Waddell DE, Oliver A, Smith DW, Fleming $\mathrm{RP}$, et al. The influence of Tai Chi training on the center of pressure trajectory during gait initiation in older adults. Arch Phys Med Rehabil. 2004;85(10):1593-8.

9. Isaias IU, Dipaola M, Michi M, Marzegan A, Volkmann J, Rodocanachi Roidi ML, et al. Gait initiation in children with Rett syndrome. PLoS One. 2014;9(4):e92736. doi: 10.1371/ journal.pone.0092736.

10. Khanmohammadi R, Talebian S, Hadian MR, Olyaei G, Bagheri $\mathrm{H}$. Characteristic muscle activity patterns during gait initiation in the healthy younger and older adults. Gait Posture. 2016;43:148-53. doi: 10.1016/j.gaitpost.2015.09.014.

11. Huang $H$, Kuiken TA, Lipschutz RD. A strategy for identifying locomotion modes using surface electromyography. IEEE Trans Biomed Eng. 2009;56(1):65-73. doi: 10.1109/ tbme.2008.2003293.

12. Bae TS, Choi K, Mun M. Level walking and stair climbing gait in above-knee amputees. J Med Eng Technol. 2009;33(2):130-5. doi: 10.1080/03091900701404043.

13. Hong JH, Mun MS. Relationship between socket pressure and EMG of two muscles in trans-femoral stumps during gait. Prosthet Orthot Int. 2005;29(1):59-72. doi: 10.1080/03093640500116764.

14. Wentink EC, Prinsen EC, Rietman JS, Veltink PH. Comparison of muscle activity patterns of transfemoral amputees and control subjects during walking. J Neuroeng Rehabil. 2013;10:87. doi: 10.1186/1743-0003-10-87.

15. Olree KS, Vaughan CL. Fundamental patterns of bilateral muscle activity in human locomotion. Biol Cybern. 1995;73(5):409-14.

16. Ivanenko YP, Poppele RE, Lacquaniti F. Five basic muscle activation patterns account for muscle activity during human locomotion. J Physiol. 2004;556(Pt 1):267-82. doi: 10.1113/ jphysiol.2003.057174

17. Hidler JM, Wall AE. Alterations in muscle activation patterns during robotic-assisted walking. Clin Biomech (Bristol, Avon). 2005;20(2):184-93. doi: 10.1016/j.clinbiomech.2004.09.016.

18. Wang L, Buchanan TS. Prediction of joint moments using a neural network model of muscle activations from EMG signals. IEEE Trans Neural Syst Rehabil Eng. 2002;10(1):30-7. doi: 10.1109/tnsre.2002.1021584.

19. Wibawa AD, Verdonschot N, Halbertsma JPK, Burgerhof JGM, Diercks RL, Verkerke GJ. A Trap Motion in Validating Muscle Activity Prediction from Musculoskeletal Model using EMG. Int J BioSci Biotechnol. 2016;8(6):61-72. doi: 10.14257/ ijbsbt.2016.8.6.07.

20. Bergmann G, Deuretzbacher G, Heller M, Graichen F, Rohlmann A, Strauss J, et al. Hip contact forces and gait patterns from routine activities. J Biomech. 2001;34(7):859-71.

21. Modenese L, Phillips ATM. Prediction of hip contact forces and muscle activations during walking at different speeds. Multibody Syst Dyn. 2012;28(1-2):157-68. doi: 10.1007/ s11044-011-9274-7.

22. Burridge $\mathrm{JH}$, Wood DE, Taylor PN, McLellan DL. Indices to describe different muscle activation patterns, identified during treadmill walking, in people with spastic drop-foot. Med Eng Phys. 2001;23(6):427-34.

23. Shao Q, Buchanan TS. A biomechanical model to estimate corrective changes in muscle activation patterns for stroke patients. J Biomech. 2008;41(14):3097-100. doi: 10.1016/j. jbiomech.2008.07.015.

24. Righetti L, Buchli J, ljspeert AJ. From Dynamic Hebbian Learning for Oscillators to Adaptive Central Pattern Generators. Switzerland: Biologically Inspired Robotics Group; 2005.

25. Taga G. Emergence of bipedal locomotion through entrainment among the neuro-musculo-skeletal system and the environment. Physica D. 1994;75(1-3):190-208. doi: 10.1016/01672789(94)90283-6.

26. Taga G. A model of the neuro-musculo-skeletal system for human locomotion. I. Emergence of basic gait. Biol Cybern. 1995;73(2):97-111.

27. Ijspeert AJ. A connectionist central pattern generator for the aquatic and terrestrial gaits of a simulated salamander. Biol Cybern. 2001;84(5):331-48. doi: 10.1007/s004220000211.

28. Collins JJ, Stewart I. Hexapodal gaits and coupled nonlinear oscillator models. Biol Cybern. 1993;68(4):287-98. doi: 10.1007/bf00201854

29. Kopell N, Ermentrout GB. Coupled oscillators and the design of central pattern generators. Math Biosci. 1988;90(1-2):87-109. doi: 10.1016/0025-5564(88)90059-4.

30. [30. Golubitsky M, Stewart I, Buono P-L, Collins JJ. A modular network for legged locomotion. Physica D. 1998;115(1-2):5672. doi: 10.1016/S0167-2789(97)00222-4.

31. Pikovsky A, Rosenblum M, Kurths J. Synchronization: A Universal Concept in Nonlinear Sciences (Cambridge Nonlinear Science Series). Cambridge, UK: Cambridge University Press; 2001:12. doi: 10.1017/CBO9780511755743.

32. Zadeh LA. The concept of a linguistic variable and its application to approximate reasoning. Inf Sci. 1975;8(3):199249. doi: 10.1016/0020-0255(75)90036-5.

33. Sabut SK, Manjunatha M, Mahadevappa M. Neuroprosthesisfunctional electrical stimulation: opportunities in clinical application for correction of drop-foot. First International Conference on Emerging Trends in Engineering and Technology; 2008:950-953. doi: 10.1109/ICETET.2008.154. 